\title{
Ophthalmological Approach to Stroke in Stroke-Prone SHR
}

\author{
Yukio Yamori, ${ }^{\circ *}$ Masako Yoshida, ${ }^{* *}$ Hidehiko Yoshida, ${ }^{* *}$ \\ and Ryoichi HoRIE ${ }^{\circ * * *}$
}

The successful establishment of stroke-prone SHR (SHRSP) has given us some important clues for both analyzing the mechanisms and exploiting the prescience and prophylaxis of stroke (Yamori et al: Clin Exp Pharmacol Physiol, 1976). For these studies, various approaches applicable to clinical investigation were attempted at our laboratory. Here methodologies such as electroencephalography and cerebral circulation were proven to be useful (Yamori et al: Jap Heart J 17: 384, 400, 1976). In the present study, we ophthalmoscopically observed retinal fundi as one of the symptomatological studies in SHRSP, and we further light-microscopically studied retinal arterioles in relation to their ophthalmoscopical findings.

\section{Materials and Methods:}

The optic fundi in 43 SHRSP, 22 stroke-resistant SHR (SHRSR) and 69 Wistar-Kyoto rats (WK) were photographed by a fundus camera (RC-II, Kowa Co, Nagoya, Japan), as reported previously (Yamori, Yoshida M, Yoshida H, Horie: In "Spontaneous Hypertension" (DHEW Publication No. (NIH) 77-1179, 1977). Their ages ranged from 2 to 17 months, and more than 20 SHRSP were in the terminal stage with neurological signs, symptoms, and general disturbances. In these SHRSP, cerebrovascular diseases (hemorrhage and infarction) were confirmed at autopsy without any exception. On the photographs of fundi in these rats at various stages, concentric circles were drawn; the center was the papilla and the diameter was 3 times the diameter of the papilla. All arterioles and venules which crossed this circle were measured to obtain the mean caliber of arterioles (mean A), the mean caliber of venules (mean V), and the mean $\mathrm{A} /$ mean $\mathrm{V}$ ratio. Eye globes were enucleated, fixed in $10 \%$ formalin solution or $95 \%$ ethylalcohol solution, and embedded in paraffin. Then the serial specimens were sectioned $5 \mu$ thick, stained with hematoxylin-eosin, and observed light-microscopically.

Results :

The following results were obtained in SHRSP. 1) In all SHRSP, narrowing and caliber-irregularity of retinal arterioles were found to some extent, being especially marked at the advanced stage. 2) In SHRSP mean A ranged from $0.042 \mathrm{~mm}$ to $0.024 \mathrm{~mm}$, and mean $V$ ranged from $0.064 \mathrm{~mm}$ to $0.061 \mathrm{~mm}$. In SHRSR mean $A$ was $0.043 \mathrm{~mm}$ and mean $V$ was $0.063 \mathrm{~mm}$. In $W K$ mean $A$ and $\mathrm{V}$ were $0.048 \mathrm{~mm}$ and $0.068 \mathrm{~mm}$, respectively. Mean $\mathrm{A} /$ mean $\mathrm{V}$ ratios in SHRSP ranged from $2 / 3$ to $2 / 5$, while those in SHRSR as well as in WK were $2 / 3$. 3) Retinal blot hemorrhage or vitreous hemorrhage was noted in some SHRSP with

From the Japan Stroke Prevention Center (JSPC), Izumo; Departments of Pathology,* Ophthalmology,** and Neurosurgery, ${ }^{* * *}$ Faculty of Medicine, Kyoto University, Kyoto. 
severe hypertension. 4) The retina was diffusely highly edematous and the optic disc also became edematous in SHRSP at the advanced stage. 5) Histologically, thickening of the subendothelial layer was noted in retinal arteriolar wall. 6) Retinal edema appeared marked in the inner layers, especially in the inner plexiform and ganglion cell layers. 7) In some SHRSP, choked disc was observed and cytoid bodies were noted in the peripapillary nerve fiber layer.

Summary with Discussion:

In SHRSP, marked hypertensive arteriolar changes were noted ophthalmoscopically and histologically, and retinal or vitreous hemorrhage was observed in some SHRSP with severe hypertension. Quantitative analyses of arteriolar and venular calibers revealed that the narrowing of arteriolar calibers was more marked in SHRSP than SHRSR. Edematous changes of retina or optic disc were often observed in SHRSP at the terminal stage. Ophthalmoscopic findings on optic fundi, also confirmed histologically in this study, are regarded as good indices showing the process of hypertensive vascular changes. Such findings may be utilized to estimate the grade of cerebrovascular lesions.

This study was supported by the Science and Technology Agency of Japanese Government, Ministry of Education, National Institutes of Health, USA (Grant HL 17754), Japan Tabacco and Salt Public Gorporation, and Japan Heart Foundation. 